Network Working Group

Request for Comments: 4481

Category: Standards Track
H. Schulzrinne Columbia U. July 2006

\title{
Timed Presence Extensions to the \\ Presence Information Data Format (PIDF) to \\ Indicate Status Information for Past and Future Time Intervals
}

Status of This Memo

This document specifies an Internet standards track protocol for the Internet community, and requests discussion and suggestions for improvements. Please refer to the current edition of the "Internet Official Protocol Standards" (STD 1) for the standardization state and status of this protocol. Distribution of this memo is unlimited.

Copyright Notice

Copyright (C) The Internet Society (2006).

Abstract

The Presence Information Data Format (PIDF) defines a basic XML format for presenting presence information for a presentity. This document extends PIDF, adding a timed status extension (<timed-status> element) that allows a presentity to declare its status for a time interval fully in the future or the past.

Table of Contents

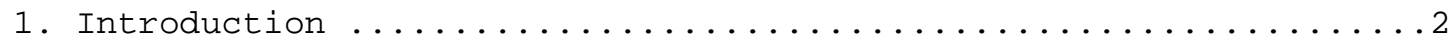

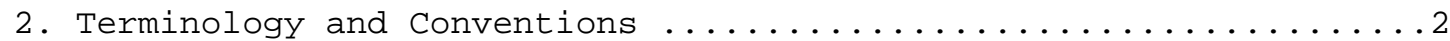

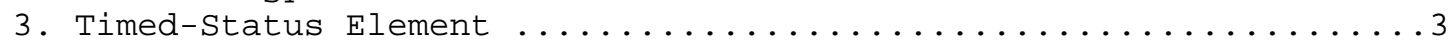

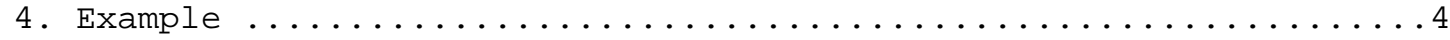

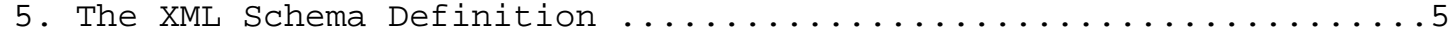

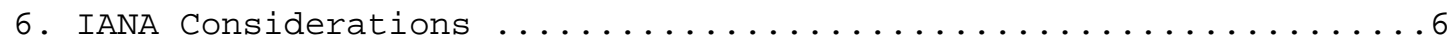

6.1. URN Sub-Namespace Registration for

' urn: ietf:params: $x m l: n s:$ pidf:timed-status' $\ldots \ldots \ldots \ldots$

6.2. Schema Registration for Schema

' urn: ietf:params: xml:ns:pidf:timed-status' $\ldots \ldots \ldots \ldots \ldots$

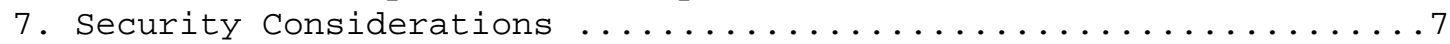

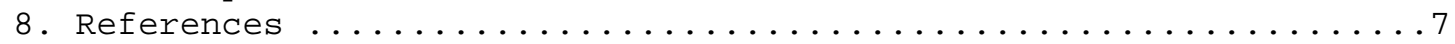

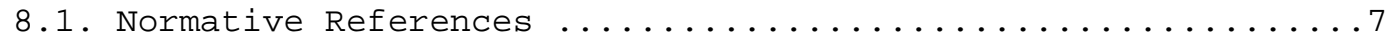

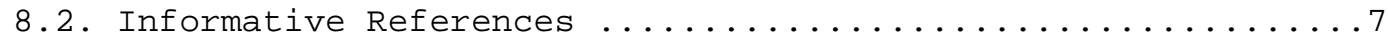

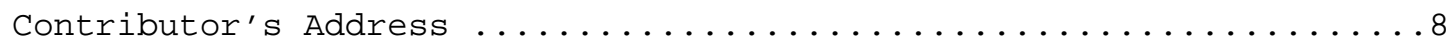

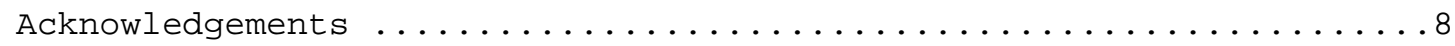




\section{Introduction}

Traditionally, presence information, e.g., represented as Presence Information Data Format [3] (PIDF) and augmented by Rich Presence Information Data format [9] (RPID), describes the current state of the presentity. However, a watcher can better plan communications if it knows about the presentity's future plans. For example, if a watcher knows that the presentity is about to travel, it might place a phone call earlier.

In this document, we use terms defined in RFC 2778 [7]. In particular, a "presentity", abbreviating presence entity, provides presence information to a presence service. It is typically a uniquely-identified person.

RPID already allows a presentity to indicate the period when a particular aspect of its presence is valid. However, the <status> element in the PIDF <tuple> does not have this facility, so that it is not possible to indicate that a presentity will be OPEN or CLOSED in the future, for example.

It is also occasionally useful to represent past information since it may be the only known presence information; it may give watchers an indication of the current status. For example, indicating that the presentity was at an off-site meeting that ended an hour ago indicates that the presentity is likely in transit at the current time.

It is unfortunately not possible to simply add time range attributes to the PIDF <status> element, as PIDF parsers without this capability would ignore these attributes and thus not be able to distinguish current from future presence status information.

This document defines the <timed-status> element that describes the status of a presentity that is either no longer valid or covers some future time period.

\section{Terminology and Conventions}

The key words MUST, MUST NOT, REQUIRED, SHOULD, SHOULD NOT, RECOMMENDED, MAY, and OPTIONAL in this document are to be interpreted as described in BCP 14, RFC 2119 [1]. 


\section{Timed-Status Element}

The <timed-status> element is a child of the <tuple> element and MUST NOT appear as a child of a PIDF <status> element or another <timed-status> element. More than one such element MAY appear within a PIDF <tuple> element.

Sources of <timed-status> information should avoid elements that overlap in time, but since overlapping appointments are common in calendars, for example, receivers MUST be able to render such overlapping <timed-status> indications.

The <timed-status> element MUST be qualified with the 'from' attribute and MAY be qualified with an 'until' attribute to describe the time when the status assumed this value and the time until which this element is expected to be valid. If the 'until' attribute is missing, the information is assumed valid until the tuple is explicitly overridden or expires as defined by the publication mechanism used. The time range MUST NOT encompass the present time, i.e., the PIDF <timestamp> value, as that would provide an unnecessary and confusing alternate mechanism to describe presence. Thus, the 'from' attribute for tuples without an 'until' attribute MUST refer to the future.

During composition, a presence agent (PA) may encounter a stored <timed-status> element that covers the present time. The PA MAY either discard that element or MAY convert it to a regular <status> element if it considers that information more credible.

The <timed-status> element may contain the <basic> and <note> elements, as well as any other element that is appropriate as a PIDF <status> extension and that has a limited validity period. Examples include the PIDF-LO [8] extensions for location objects.

This extension chose absolute rather than relative times, since relative times would be too hard to keep properly updated when spacing notifications, for example. Originators of presence information MUST generate time values in the <timed-status> elements that are fully in the past or future relative to local real (wallclock) time and the time information contained in the optional PIDF <timestamp> element. 
4. Example

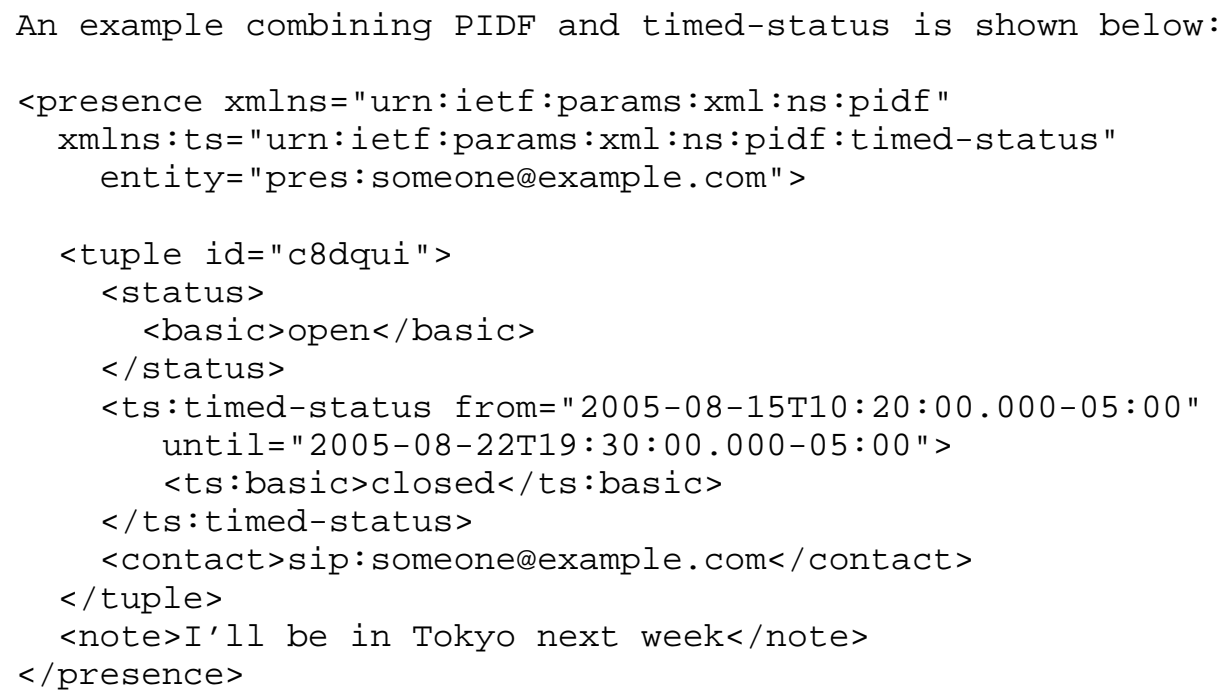


5. The XML Schema Definition

The XML [4] schema [5] [6] is shown below.

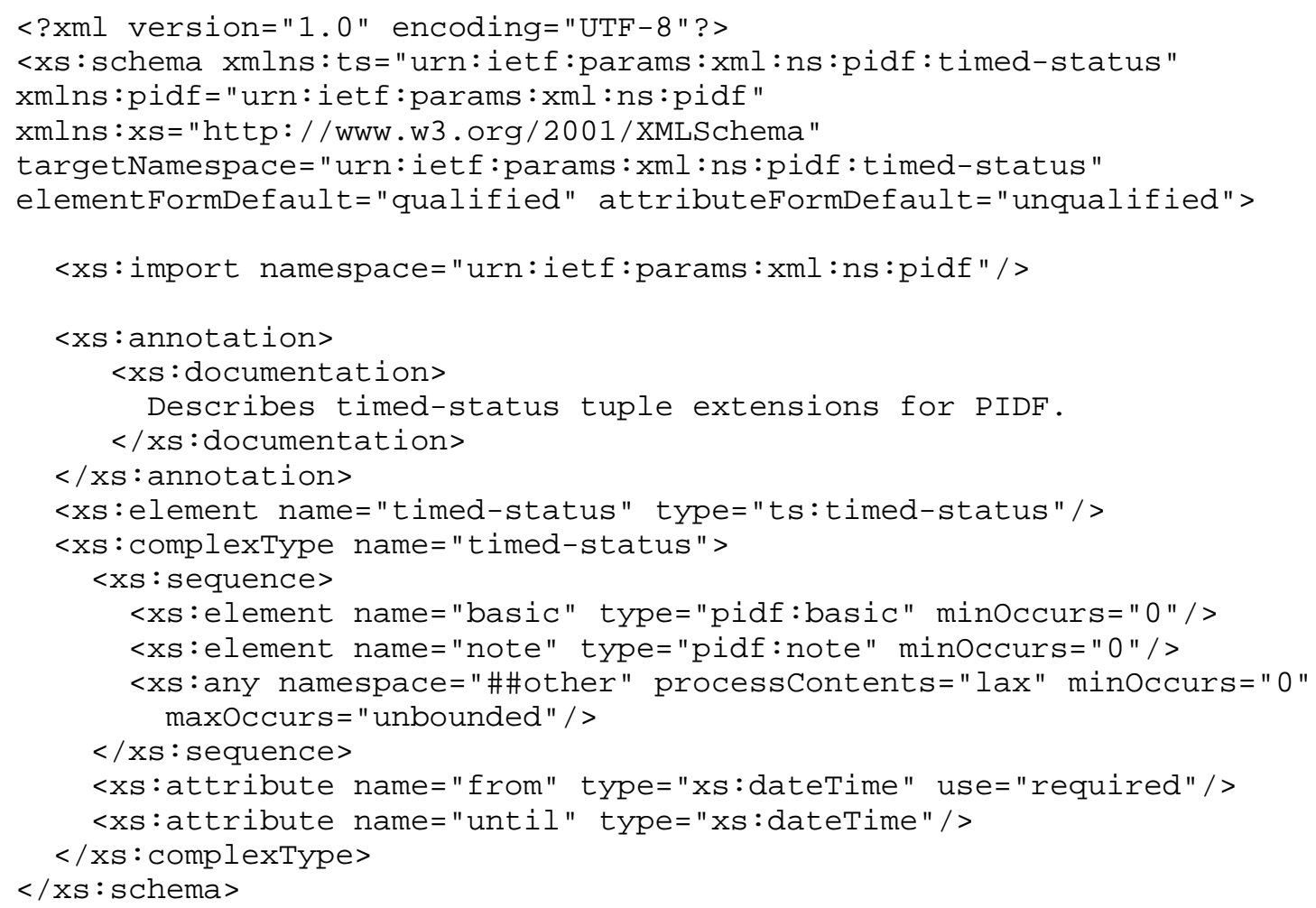


6. IANA Considerations

This document calls for IANA to register a new XML namespace URN and schema per [2].

6.1. URN Sub-Namespace Registration for

' urn: ietf:params:xml:ns:pidf:timed-status'

URI: urn:ietf:params:xml:ns:pidf:timed-status

Description: This is the XML namespace for XML elements defined by RFC 4481 to describe timed-status presence information extensions for the status element in the PIDF presence document format in the application/pidf+xml content type.

Registrant Contact: IETF, SIMPLE working group, simpledietf.org; Henning Schulzrinne, hgs@cs.columbia.edu

XML :

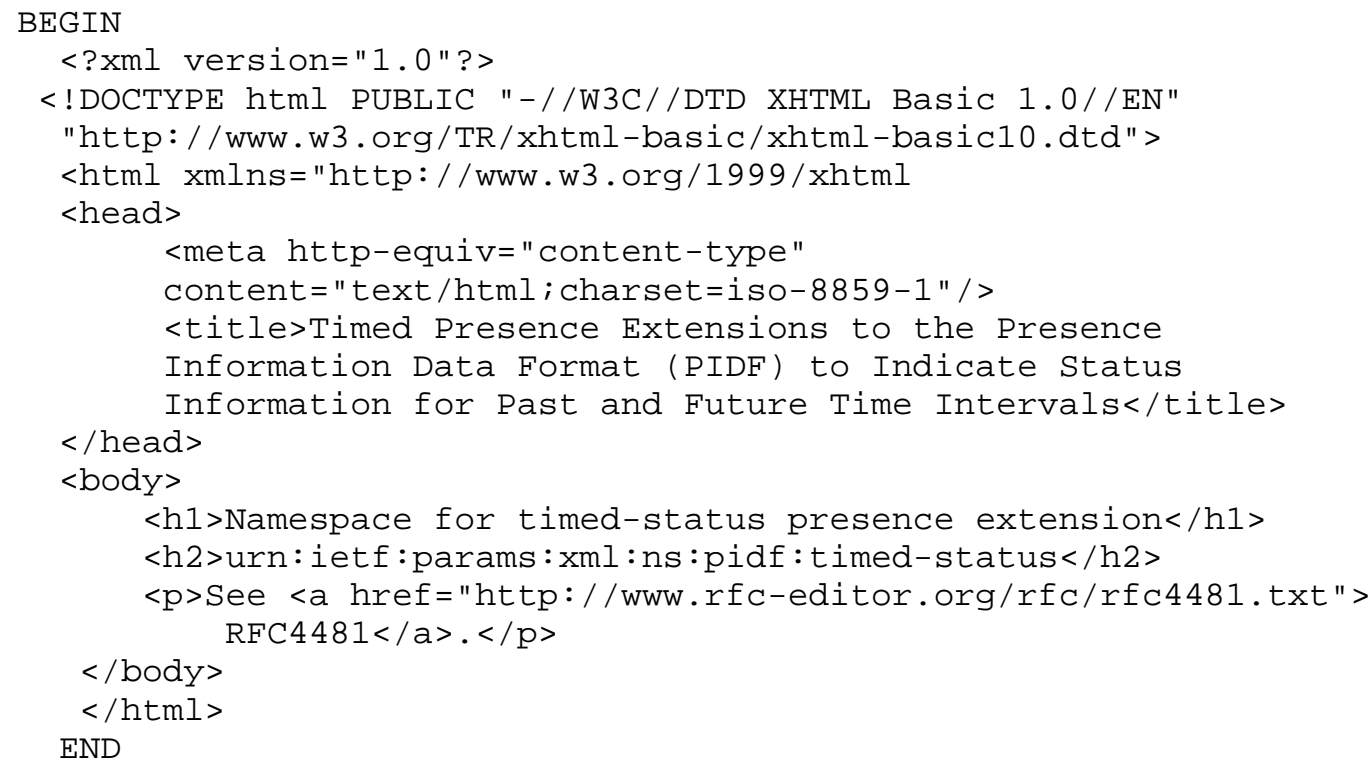


6.2. Schema Registration for Schema

' urn: ietf:params: xml:ns:pidf:timed-status'

URI: urn:ietf:params:xml:ns:pidf:timed-status

Registrant Contact: IESG

XML: See Section 5

7. Security Considerations

The security issues are similar to those for RPID [9].

8. References

8.1. Normative References

[1] Bradner, S., "Key words for use in RFCs to Indicate Requirement Levels", BCP 14, RFC 2119, March 1997.

[2] Mealling, M., "The IETF XML Registry", BCP 81, RFC 3688, January 2004 .

[3] Sugano, H., Fujimoto, S., Klyne, G., Bateman, A., Carr, W., and J. Peterson, "Presence Information Data Format (PIDF)", RFC 3863, August 2004 .

[4] Yergeau, F., Paoli, J., Sperberg-McQueen, C., Bray, T., and E. Maler, "Extensible Markup Language (XML) 1.0 (Third Edition)", W3C REC REC-xml-20040204, February 2004.

[5] Maloney, M., Beech, D., Thompson, H., and N. Mendelsohn, "XML Schema Part 1: Structures Second Edition", W3C REC RECxmlschema-1-20041028, October 2004 .

[6] Malhotra, A. and P. Biron, "XML Schema Part 2: Datatypes Second Edition", W3C REC REC-xmlschema-2-20041028, October 2004.

8.2. Informative References

[7] Day, M., Rosenberg, J., and H. Sugano, "A Model for Presence and Instant Messaging", RFC 2778, February 2000.

[8] Peterson, J., "A Presence-based GEOPRIV Location Object Format", RFC 4119, December 2005. 
[9] Schulzrinne, H., Gurbani, V., Kyzivat, P., and J. Rosenberg, "RPID: Rich Presence Extensions to the Presence Information Data Format (PIDF)", RFC 4480, July 2006.

Contributor's Address

Jonathan Rosenberg

dynamicsoft

600 Lanidex Plaza

Parsippany, NJ 07054-2711

USA

EMail: jdrosen@dynamicsoft.com

Acknowledgements

This document is based on the discussions within the IETF SIMPLE

working group. Mary Barnes, Avri Doria, Miguel Garcia, Vijay

Gurbani, Hisham Khartabil, Paul Kyzivat, Mikko Lonnfors, Yannis

Pavlidis and Jon Peterson provided helpful comments.

Author's Address

Henning Schulzrinne

Columbia University

Department of Computer Science

450 Computer Science Building

New York, NY 10027

US

Phone: +1 2129397004

EMail: hgstsimple@cs.columbia.edu

URI: http://www.cs.columbia.edu 
Full Copyright statement

Copyright (C) The Internet Society (2006).

This document is subject to the rights, licenses and restrictions contained in BCP 78, and except as set forth therein, the authors retain all their rights.

This document and the information contained herein are provided on an "AS IS" basis and THE CONTRIBUTOR, THE ORGANIZATION HE/SHE REPRESENTS OR IS SPONSORED BY (IF ANY), THE INTERNET SOCIETY AND THE INTERNET ENGINEERING TASK FORCE DISCLAIM ALL WARRANTIES, EXPRESS OR IMPLIED, INCLUDING BUT NOT LIMITED TO ANY WARRANTY THAT THE USE OF THE INFORMATION HEREIN WILL NOT INERINGE ANY RIGHTS OR ANY IMPLIED WARRANTIES OF MERCHANTABILITY OR FITNESS FOR A PARTICULAR PURPOSE.

Intellectual Property

The IETF takes no position regarding the validity or scope of any Intellectual Property Rights or other rights that might be claimed to pertain to the implementation or use of the technology described in this document or the extent to which any license under such rights might or might not be available; nor does it represent that it has made any independent effort to identify any such rights. Information on the procedures with respect to rights in RFC documents can be found in BCP 78 and BCP 79 .

Copies of IPR disclosures made to the IETF Secretariat and any assurances of licenses to be made available, or the result of an attempt made to obtain a general license or permission for the use of such proprietary rights by implementers or users of this specification can be obtained from the IETF on-line IPR repository at http://www.ietf.org/ipr.

The IETF invites any interested party to bring to its attention any copyrights, patents or patent applications, or other proprietary rights that may cover technology that may be required to implement this standard. Please address the information to the IETF at ietf-ipreietf.org.

Acknowledgement

Funding for the RFC Editor function is provided by the IETF Administrative Support Activity (IASA). 